\title{
Estudio descriptivo de los resultados del Programa Mamá Canguro del Hospital de Ia Mujer, Ags.
}

\author{
Díaz-Martínez Roberto Moisés*, Acevedo-Ruíz Esparza Blanca Azucena*, Monroy-Favela Lilibeth*
}

\section{Resumen}

- El Método Mamá Canguro (MMC), se caracteriza porque - mantiene en contacto piel a piel al neonato con su madre, - son alimentados con la leche materna y promueve un egre-

- so temprano del hospital. Es un método fácil de aplicar, que

- fomenta la salud y el bienestar tanto de los recién nacidos

- prematuros como de los nacidos a término. Nuestro objetivo

- fue conocer las características generales de los neonatos del

- Programa Mamá Canguro (PMC) del Hospital de la Mujer. Se

- realizó un estudio descriptivo, retrospectivo, y transversal. Se re-

- visaron 71 casos de pacientes del Programa Mamá Canguro

- del Hospital de la Mujer entre marzo de 2009 y agosto de

- 2010, mediante un muestreo por conveniencia. Encontramos

- que el promedio de edad gestacional al nacimiento fue de 32

- \pm 2.4 semanas, el peso promedio al ingresar al PMC fue de

- $1400 \pm 204 g$, el promedio del peso al egreso del PMC fue de

- $1754 \pm 185 g$, con una ganancia de peso promedio a través

- del MMC, de 22.1 g/día. Predominó la interrupción del em-

- barazo mediante operación cesárea en el 82\% de los casos y

- un poco más de la mitad de los paciente requirieron de ma-

- niobras de reanimación neonatal. La mayoría de los pacientes

- fueron alimentados con fórmula para prematuros combinada

- con leche materna. El 87.3\% de los neonatos, egresaron del

- servicio por mejoría. El PMC es una buena alternativa en la

- atención de los productos pretérmino. En el Hospital de la

- Mujer se debe promover el incremento del uso de leche ma-

- terna. LUX MÉDICA, AÑ0 8, NÚMERO 25, SEPTIEMBRE-DICIEMBRE

2013,PP 55-65

\section{Abstract}

The Mama Kangaroo Method (MKM), stands out because it keeps in contact skin-to-skin to the infant with his mother, they are fed with breast milk and promotes an early discharge from the hospital. It is an easy-to-apply method, which promotes health and well-being both of the preterm birth and of those born at term. Our objective was to understand the general characteristics of infants of Mama Kangaroo Method (MKM) of the Women's Hospital. A descriptive, retrospective, and cross- sectional study. Were reviewed 7 I cases of patients of Mama Kangaroo Method of the Women's Hospital, between March 2009 to August 2010, through sampling by convenience. We found that the average gestational age at birth was 32 \pm 2.4 weeks, the average weight to join the MKM was 1400 $\pm 204 \mathrm{~g}$, average weight at the end of the MKM was 1754 \pm 185 grams, with a gain of average weight through (MKM), $22.1 \mathrm{~g} /$ day. The Predominated the termination of pregnancy through caesarean in $82 \%$ of cases and a little more than half of the patient required neonatal resuscitation maneuvers. Most of the patients were fed with formula for preterm combined with breast milk The $87.3 \%$ of infants out from service for improvement. The MKM is a good alternative in the care of the preterm products. The increase in the use of breastfeeding should be promoted at the Women's Hospital. LUX MÉDICA, AÑ0 8, NÚMERO 25, SEPTIEMBRE-DICIEMBRE 2013, PP 55-65

Estudiantes del noveno semestre de Medicina de la Universidad Autónoma de Aguascalientes

Fecha de recibido: 9 de abril 2013

Fecha de aceptación: 9 de octubre 2013

Correspondencia: Dra Ma del Carmen Terrones Saldívar, Jefa del Depto de Gineco-Obstetricia y Pediatría del Centro de Ciencias de la Salud de la Universidad Autónoma de Aguascalientes. Avenida Universidad 940, Edificio 107, planta alta. Ciudad Universitaria CP20131, Aguascalientes, Ags., México teléfono 01(449)9108443. Correo electrónico mcterron@correo.uaa.mx 


\section{Introducción}

A pesar de los adelantos logrados por la neonatología del siglo $X X$, al disminuir las cifras de morbimortalidad perinatal, el bajo peso al nacer (menos de 2000 g) y la prematurez, continúan presentándose como un gran problema de salud pública. ${ }^{1,2}$ Cada año, en el mundo nacen cerca de 20 millones de niños prematuros o con bajo peso al nacer, la mayoría en países poco desarrollados, con recursos insuficiente para limitar la posibilidad de llevar a cabo intervenciones eficaces, por lo tanto se mantienen elevada la tasa de mortalidad neonatal. ${ }^{3}$ Se estima que anualmente se presentan 4 millones de muertes neonatales, de éstas, los recién nacidos prematuros representan cerca del $20 \%$. Por lo anterior, además de la pérdida de vidas, el nacimiento prematuro representa costos económicos y emocionales considerables para las familias y las comunidades..$^{1,4} \mathrm{En}$ países en desarrollo, las causas que producen el parto pretérmino disminuyen lentamente, ocasionando tasas elevadas de nacimientos prematuros y neonatos con bajo peso al nacer. ${ }^{5}$

Para responder a la necesidad de disminuir las muertes neonatales en especial en los países en vías de desarrollo, se propuso la intervención denominada Método de Mamá Canguro (MMC) que dió inicio en 1978, cuando los doctores Rey y Martínez propusieron y desarrollaron este método en el Instituto Materno Infantil de Santa Fe de Bogotá, Colombia, como una alternativa al método convencional de atención para neonatos prematuros y de bajo peso al nacer. Las madres se utilizan como "incubadoras" y como fuente principal de alimento y estimulación para los neonatos hasta que alcanzan la maduración suficiente para enfrentar la vida extrauterina en condiciones similares a las de aquellos nacidos a término. ${ }^{1}$ Esta metodología, se utiliza en más de 48 países incluido México. Debido a su utilidad y a su extensión en todo el mundo, la UNICEF ha acompañado y apoyado el desarrollo del MMC. ${ }^{6}$ Es así, como después de dos décadas de aplicación e investigación, fue aceptado como una alternativa para el manejo del recién nacido prematuro, tratándose de un complemento del cuidado neonatal. ${ }^{7}$ Existen autores que han reportado que el principal impacto del $M M C$ es la protección neurológica ${ }^{5,8-10}$; además de asociarse con la disminución en la mortalidad neonatal, la estancia hospitalaria, y severidad de infecciones.

Otras ventajas del MMC son las siguientes: mejor regulación de la temperatura corporal del neonato, ${ }^{11-13}$ mejoría en los patrones reguladores de respiración, con una disminución de episodios de apnea, ${ }^{12-14}$ estados de comportamiento del bebé más regulados, ${ }^{13}$ disminución de severidad de infecciones, infección nosocomial y sepsis, ${ }^{12,13,15-17}$ efecto positivo en el crecimiento y desarrollo neonatal, ${ }^{12,16,18-23}$ mayor aceptabilidad y éxito en la lactancia, ${ }^{12,16,22,24}$ disminución del estrés en el neonato, ${ }^{25}$ reducción de la morbilidad y mortalidad neonatal, ${ }^{12,15-17}$ aumenta los periodos de sueño. ${ }^{12,13}$ 
Con respecto a los padres: en las madres, existe más confianza en sí mismas, sentimiento de realización, menos estrés, y depresión, ${ }^{12,26}$ y favorece un mayor vínculo entre madre e hijo. 9,13,27,28

Si consideramos que la Organización Mundial de la Salud (OMS) reconoce a la lactancia materna como una prioridad nutricional mundial, entonces es fácil comprender que el prematuro y el recién nacido con bajo peso deben iniciar con la lactancia lo más pronto posible y a libre demanda. Si el neonato aun no tiene reflejo de succión/ deglución adecuada se inicia la alimentación con sonda nasogástrica, jeringa o gotero. ${ }^{1,4}$ Tras la pérdida de peso inicial, los neonatos recobran lentamente peso. No hay límite superior para el aumento de peso de recién nacidos alimentados con leche materna, mientras que el límite inferior no debe en ningún caso quedar por debajo de los $15 \mathrm{~g} /$ día. 4,22 En nuestro estado no existe información acerca de este programa, por lo que nuestro objetivo fue describir las características generales de los neonatos ingresados al Programa de Mamá Canguro (PMC) del Hospital de la Mujer de Aguascalientes.

| | | | | | | | | | | | | | | | | | | | | | | | | | | | | | | | | | | | | | | | | | | | | | | | | | | | | | | | | | | | | | | | | | | | | | | | | | | | | | | | | | | | | | | | | | | | | | | | | | | | | | | | | | | | | | | | | | | | |

\section{Material y métodos}

Se realizó un estudio descriptivo, retrospectivo y transversal. Se obtuvieron los expedientes de los recién nacidos menores de 36 semanas de gestación que ingresaron al Programa Mamá Canguro del Hospital de la Mujer en Aguascalientes entre marzo de 2009 y agosto de 2010. El muestreo fue por conveniencia. Se incluyeron para el estudio un total de 71 casos. Las variables que se recabaron fueron: edad gestacional al nacimiento, peso al nacimiento, motivo del nacimiento prematuro, patologías asociadas, enfermedades maternas, edad y escolaridad materna, nivel socioeconómico, peso de ingreso al $M M C$, peso de egreso al MMC y el tipo de alimentación durante el programa. Los resultados se informan en medidas de tendencia central.

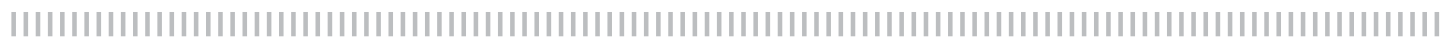

\section{Resultados}

1. Datos de los neonatos: Se estudiaron 71 recién nacidos pretérmino del Programa Mamá Canguro. Todos los neonatos fueron clasificados como pretérmino (32 \pm 2.4 semanas de gestación, con un rango de 22 a 36 semanas). Predominó el sexo femenino con una frecuencia de $63.3 \%(n=45)$, al sexo masculino le correspondió el $36.6 \%(n=26)$. El promedio de la talla fue de $40.2 \pm 7 \mathrm{~cm}$ y el del perímetro cefálico fue de $28.1 \pm 2.5 \mathrm{~cm}$. El
$19.71 \%$ de los neonatos $(n=14)$ fueron prematuros leves, (edad gestacional de 34 a 36 semanas), el $45.07 \%(n=32)$ fueron prematuros moderados (32 a 33 SDG), mientras que el $29.57 \%(n=21)$ tuvieron prematurez severa, (28 a 31SDG) y sólo un $5.63 \%(n=4)$ fueron clasificados con prematurez extrema (20 a 27 SDG). 25 (Tabla 1). En cuanto a la calificación de Apgar, los neonatos tuvieron en promedio $7.4 \pm 1.4$ al minuto y $8.5 \pm 0.6$ a los cinco minutos. 


\section{Tabla I}

\section{Distribución y frecuencia de los neonatos prematuros del Programa Mamá Canguro, según su} edad gestacional.

\begin{tabular}{|lcc|}
\hline Semanas de Gestación & $\begin{array}{c}\text { Número } \\
(\mathrm{n}=71)\end{array}$ & $\begin{array}{c}\text { Porcentaje } \\
(\mathrm{n}=71)\end{array}$ \\
\hline Prematurez leve (34-36) & 14 & 19.7 \\
\hline Prematurez moderada (32-33) & 32 & 45.1 \\
\hline Prematurez severa 28-31 & 21 & 29.5 \\
\hline Prematurez extrema $<28$ & 4 & 5.7 \\
\hline
\end{tabular}

Según su peso al nacer, encontramos que el promedio fue de 1,388 $\pm 302 \mathrm{~g}$, (rango de 475 a 1,900 g). En orden decreciente en frecuencia, observamos al grupo de peso muy bajo al nacimiento, con 33 neonatos $(46.5 \%)$, seguido de los neonatos con bajo peso al nacimiento en 30 de los casos $(42.3 \%)$ y al grupo de peso extrema- damente bajo $(<1,000 \mathrm{~g})$ pertenecieron 8 casos $(11.2 \%)$. Al momento de ingresar al PMC, siguió predominando el neonato con muy bajo peso al nacer, seguido del neonato con bajo peso al nacer y por último el recién nacido con peso extremadamente bajo, con una frecuencia de $60.5 \%, 36.7 \%$ y $2.8 \%$, respectivamente (tabla 2 ).

\section{Tabla 2}

Distribución y frecuencia de los neonatos prematuros según su peso al nacer y el peso al ingresar al Programa Mamá Canguro.

\begin{tabular}{|l|c|c|c|c|}
\hline \multirow{2}{*}{ Peso (gramos) $(\mathrm{n}=\mathbf{7 1})$} & \multicolumn{2}{c|}{ Número } & \multicolumn{2}{c|}{ Porcentaje } \\
& Al nacer & Al ingresar al PMC & Al nacer & Al ingresar al PMC \\
\hline $\mathbf{1 5 0 0 - 2 5 0 0}$ & 30 & 26 & 42.3 & 36.7 \\
\hline $\begin{array}{l}\text { Recién nacido con bajo } \\
\text { peso al nacer }\end{array}$ & & & & \\
\hline $\mathbf{1 0 0 0 - 1 4 9 9}$ & 33 & 43 & 46.5 & \\
\hline $\begin{array}{l}\text { Recién nacido con muy } \\
\text { bajo peso al nacer }\end{array}$ & & & & \\
\hline$<\mathbf{1 0 0 0}$ & 8 & 2 & 11.2 & \\
\hline $\begin{array}{l}\text { Recién nacido con extre- } \\
\text { madamente bajo peso al nacer }\end{array}$ & & & & \\
\hline
\end{tabular}

Como podemos observar en la tabla 3, el peso promedio de los neonatos al ingresar al Programa de Mamá Canguro fue de $1,400 \pm 204 \mathrm{~g}$, con un rango entre $930 \mathrm{y}$ $1,770 \mathrm{~g}$, mientras que a su egreso fue de $1754 \pm 185 \mathrm{~g}$, con un rango de 1,300$2,100 \mathrm{~g}$ (figura 1). El tiempo neto de hospitalización de estos pacientes desde su nacimiento hasta su egreso fue de $29.5 \pm 14.2$ (rango de 8 a 62 días). Ya ingresados en el
PMC, el promedio de ganancia ponderal de los neonatos fue de $344 \pm 241 \mathrm{~g}$ (rango de 30 a 990 g) durante un promedio de estancia hospitalaria $15.1 \pm 8.8$ días.

El tipo de alimentación más frecuente en el PMC fue la combinación del leche materna y fórmula para prematuros (49.2\%), seguido del uso de fórmula para prematuro $(40.8 \%)$, la lactancia materna exclusiva en un $(8.5 \%)$, y el uso de fórmula de soya solo 
para casos excepcionales en un (1.4\%). El ingresado o reingresado al servicio de Cune$87.3 \%$ de los paciente $(n=62)$ fueron da- ro Patológico I (CUPA I) y un $2.8 \%(n=2)$ al dos de alta por mejoría, un $9.9 \%(n=7)$ fue servicio de Cunero Patológico II (CUPA II).

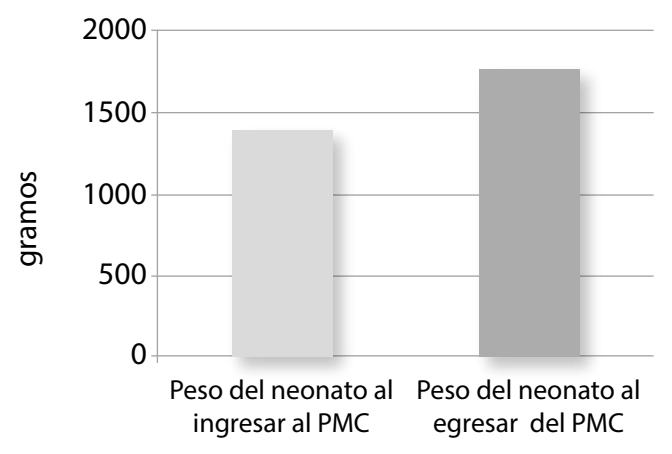

Figura 1. Promedio de los pesos (en gramos) de los neonatos, al ingreso y al egreso del Programa Mamá Canguro.

\section{Tabla 3}

Distribución y frecuencia de algunos parámetros en los prematuros del Programa Mamá Canguro.

\begin{tabular}{|c|c|}
\hline \multicolumn{2}{|l|}{$\begin{array}{l}\text { Características de los neonatos ingresados al Programa } \\
\text { Mamá Canguro }(n=71)\end{array}$} \\
\hline $\begin{array}{l}\text { Promedio del peso al ingreso (g) } \\
\text { (rango) }\end{array}$ & $\begin{array}{l}1400 \pm 204 \\
(930-1770)\end{array}$ \\
\hline $\begin{array}{l}\text { Promedio del peso al egreso (g) } \\
\text { (rango) }\end{array}$ & $\begin{array}{c}1754 \pm 185 \\
(1,300-2,100)\end{array}$ \\
\hline Promedio de ganancia de peso (g) & $\begin{array}{c}344 \pm 241 \\
(30-990)\end{array}$ \\
\hline Días de estancia hospitalaria (días) & $\begin{array}{l}15.1 \pm 8.8 \\
(4-40)\end{array}$ \\
\hline Lactancia materna + fórmula para prematuros & $\begin{array}{c}35 \\
(49.2 \%)\end{array}$ \\
\hline Tipo de alimentación (n,\%) & $\begin{array}{c}29 \\
(40.8 \%)\end{array}$ \\
\hline Lactancia materna exclusiva & $\begin{array}{c}6 \\
8.5 \%\end{array}$ \\
\hline Fórmula de soya & $\begin{array}{c}1 \\
1.5 \%\end{array}$ \\
\hline
\end{tabular}

\section{Datos maternos}

La edad materna promedio fue de $25 \pm 7$ años en un rango de 14 a 40 años. En el $40.8 \%(n=29)$ de los casos, la edad de la madre fue considerada de riesgo, ya sea menor de 19 años (31\%, $\mathrm{n}=22)$ o mayor de 35 años $(9.8 \%, n=7)$, el resto de las pacientes $(59.2 \%, n=42)$ se encontraron 
en un rango ideal para la concepción. En orden decreciente, la escolaridad materna fue la siguiente: secundaria completa $(43.7 \%, \mathrm{n}=31))$, primaria completa $(32.4 \%, n=23)$, preparatoria completa $(7 \%, n=5)$, secundaria incompleta $(5.6 \%$, $\mathrm{n}=4)$, preparatoria incompleta $(5.6 \%$, $n=4)$, licenciatura $(2.8 \%, n=2)$, primaria incompleta $(1.4 \%, n=1)$ y carrera técnica $(1.4 \%, n=1)$.

La distribución del estado civil entre las madres estudiadas mostró que la mayoría refirió estar casada $(42.3 \%, n=30)$, seguida de la unión libre $(36.6 \%, n=26)$ y soltera en el $21.2 \%(n=15)$ de los casos. Casi la totalidad de las madres refirieron estar dedicadas a las labores del hogar (94.4\%, $n=67)$ y un $5.6 \%(n=4)$ trabajaban como empleadas.

El consumo de sustancias tóxicas se presentó en un $13 \%(n=9)$ de las madres de las cuales consumieron durante parte o todo el embarazo una o más sustancias de las siguientes: tabaco (88.8\%), alcohol $(33.3 \%)$, marihuana $(11.1 \%)$ y cocaína (11.1\%).

En cuanto a la paridad, encontramos que predominó la primigesta en un $40.8 \%$ $(n=29)$, seguida de la multípara $(n=26$, $36.6 \%$ ), la secundigesta en el $21.2 \%$ $(n=15)$ y una gran multípara (1.4\%).

Las madres presentaron alguna, varias o ninguna patología durante el embarazo como muestra la tabla 4 .

\section{Tabla 4}

Morbilidad asociada al embarazo de las madres de los neonatos del PMC.

\begin{tabular}{|c|c|c|}
\hline PATOLOGIA O INTERVENCION & Número & Porcentaje \\
\hline Infección de vías urinarias & 39 & 54.9 \\
\hline Cervicovaginitis & 22 & 31 \\
\hline Hipertensión arterial & 6 & 8.5 \\
\hline Preeclampsia & 6 & 8.5 \\
\hline Amenaza de aborto & 6 & 8.5 \\
\hline Diabetes gestacional & 4 & 5.6 \\
\hline Hemorragia en el primer trimestre & 4 & 5.6 \\
\hline Hemorragia de la segunda mitad del embarazo & 2 & 2.8 \\
\hline Cerclaje & 2 & 2.8 \\
\hline Corioamnioitis & 2 & 2.8 \\
\hline Síndrome de HELLP & 2 & 2.8 \\
\hline Eclampsia & 1 & 1.4 \\
\hline Neumonía & 1 & 1.4 \\
\hline Fiebre & 1 & 1.4 \\
\hline Rh - & 1 & 1.4 \\
\hline Ninguna & 12 & 16.9 \\
\hline
\end{tabular}


El equipo de salud tomó la decisión en el $82 \%$ de los casos, de interrumpir el embarazo por vía abdominal y en el $18 \%$ de los casos, se atendieron eutocias. Las razones reportadas en el expediente clínico por las cuales se tomó la decisión de cesárea o parto se reportan en la tabla 5 .

\section{Tabla 5}

Patología de la madre al momento de la finalización del embarazo.

\begin{tabular}{|c|c|c|c|c|}
\hline PATOLOGIA & CESAREA & $(n=58)$ & PARTO \% & $(n=13)$ \\
\hline Ruptura prematura de membranas & 17 & $(29.3 \%)$ & 6 & $(46.2 \%)$ \\
\hline Oligohidramnios & 9 & $(15.5 \%)$ & - & \\
\hline Sufrimiento fetal & 7 & $(12.1 \%)$ & 1 & $(7.7 \%)$ \\
\hline Embarazo gemelar & 7 & $(12.1 \%)$ & - & \\
\hline Desprendimiento prematuro de placenta & 7 & $(12.1 \%)$ & - & \\
\hline Trabajo de parto pretérmino & 6 & $(10.3 \%)$ & 1 & $(7.7 \%)$ \\
\hline Placenta previa & 4 & $(6.9 \%)$ & - & \\
\hline Preeclampsia & 4 & $(6.9 \%)$ & 1 & $(7.7 \%)$ \\
\hline Presentación pélvica & 3 & $(5.2 \%)$ & 1 & $(7.7 \%)$ \\
\hline Trabajo de parto inminente & 2 & $(3.4 \%)$ & 4 & $(30.8 \%)$ \\
\hline Retraso del crecimiento intrauterino & 2 & $(3.4 \%)$ & - & \\
\hline Cesárea previa & 2 & $(3.4 \%)$ & - & \\
\hline Inductoconducción fallida & 1 & $(1.7 \%)$ & - & \\
\hline Eclampsia & 1 & $(1.7 \%)$ & - & \\
\hline Presentación transversa & 1 & $(1.7 \%)$ & - & \\
\hline Prolapso de membranas & 1 & $(1.7 \%)$ & - & \\
\hline Periodo intergenésico corto & 1 & $(1.7 \%)$ & - & \\
\hline $\mathrm{Pb}$. sepsis & 1 & $(1.7 \%)$ & - & \\
\hline Insuficiencia cervical & 1 & $(1.7 \%)$ & - & \\
\hline Cesárea Iterativa & 1 & $(1.7 \%)$ & - & \\
\hline Parto fortuito & - & & 2 & $(15.4 \%)$ \\
\hline Sin ninguna indicación escrita & 1 & $(1.7 \%)$ & & \\
\hline
\end{tabular}

Un 52\% de los neonatos requirió de medidas extras de reanimación como el oxígeno suplementario (38\%), ventilación a presión positiva $(11 \%)$, dos o más ciclos de presión positiva ( $8 \%$ ), intubación (35\%), catéter umbilical (3\%), y un $5 \%$ sufrió de trauma obstétrico además de alguna de las maniobras anteriormente listadas. Después del nacimiento todos los productos presentaron alguna patología o condición como muestra la tabla 4. 


\section{Tabla 6}

\section{Diagnósticos de los productos al nacer.}

\begin{tabular}{|c|c|c|}
\hline PATOLOGIA (n=71) & Número & Porcentaje \\
\hline Síndrome de distress respiratorio & 61 & 85.9 \\
\hline Hiperbilirrubinemia multifactorial & 34 & 47.9 \\
\hline Sepsis o probable sepsis & 12 & 16.9 \\
\hline Retraso del crecimiento intrauterino & 7 & 9.9 \\
\hline Displasia broncopulmonar & 7 & 9.9 \\
\hline Enfermedad de membrana hialina & 6 & 8.5 \\
\hline Hipoglucemia & 3 & 4.2 \\
\hline Persistencia del conducto arterioso & 2 & 2.8 \\
\hline Hemorragia intraventricular & 1 & 2.8 \\
\hline Neumonía in útero & 1 & 1.4 \\
\hline Síndrome de Down & 1 & 1.4 \\
\hline Dismorfias & 1 & 1.4 \\
\hline Hemorragia pulmonar & 1 & 1.4 \\
\hline Rh negativo & 1 & 1.4 \\
\hline Atelectasia & 1 & 1.4 \\
\hline Apneas mixtas & 1 & 1.4 \\
\hline Desnutrición in útero & 1 & 1.4 \\
\hline VIH & 1 & 1.4 \\
\hline
\end{tabular}

\section{Discusión}

En este trabajo, se revisaron 71 expedientes de neonatos prematuros que ingresaron al programa MMC del Hospital de la Mujer. El peso promedio al nacer fue de $1,388 \pm 302 \mathrm{~g}(475 \mathrm{a} 1,900 \mathrm{~g})$, el peso promedio al ingresar al MMC fue de 1400 \pm 204 (930-1770), y el peso al momento de egreso fue en promedio de $1754 \pm 185$ $(1,300-2,100)$. La mayoría de los pacientes fueron dados de alta por mejoría posterior a una estancia hospitalaria promedio de 15 \pm 9 días. Si comparamos nuestros resultados con los reportados por Lizarazo y cols (2012), observamos que ellos encontraron mayor peso al nacer en los 374 recién nacidos prematuros que estudiaron, de $1969 \mathrm{~g}$ vs $1,388.7 \mathrm{~g}$ en nuestro estudio; en cuanto al peso de egreso, aquellos reportaron un promedio de 2,742 g vs 1753.7 en nuestro estudio. El promedio de ganancia ponderal encontrado por nosotros fue $22.1+/$ $7.4 \mathrm{~g} /$ día, similar al grupo de Lizarazo (22 g). ${ }^{29}$

Los tres elementos básicos del programa MMC son la lactancia materna, el contacto piel a piel en posición canguro y el egreso temprano al hogar. La Organización Mundial de la Salud emitió una guía práctica sobre el Método Mamá Canguro en el año de 2004, después de analizar la evidencia científica disponible. El MMC es la atención a los niños prematuros manteniéndolos en contacto piel a piel con su madre. Se trata de un método eficaz y fácil que fomenta la salud y el bienestar tanto de los recién nacidos prematuros como de los nacidos a término. ${ }^{30}$ En este estudio descriptivo, en- 
contramos que todos los neonatos fueron pretérmino y abarcaron un rango desde pretérminos leves hasta prematuros extremos. Las dos causas más frecuente del parto pretérmino, de acuerdo a estos resultados, fueron la infección de vías urinarias en más de la mitad de los casos, (54.9\%), seguido de la cervicovaginitis (31\%). En menor frecuencia encontramos los estados hipertensivos del embarazo, la diabetes gestacional y las hemorragias de la segunda mitad del embarazo, entre otras. El médico de primer contacto debe llevar un control prenatal adecuado, con la intención de identificar tempranamente las dos causas más frecuentes asociadas al parto pretérmino, como ya lo mencionamos, la urosepsis y la cervicovaginitis. Otros factores que se asocian al parto pretérmino, es la edad materna en los extremos de la vida reproductora: en este estudio, la edad materna de riesgo ${ }^{31}$ estuvo identificada en cuatro de cada diez madres de neonatos pretérminos $(40.8 \%)$. Siendo éste un factor de riesgo que puede ser controlado, se debe promover reducir los embarazos en mujeres adolescentes o en mujeres tardías. Las toxicomanías también están asociadas al parto pretérmino, nosotros encontramos que un poco más de una de cada diez madres refirieron tabaquismo, ingirieron alcohol, fumaron mariguana o consumieron cocaína. Si bien no es elevada la prevalencia de esas toxicomanías, evitando su consumo durante la gestación, se estará disminuyendo los riesgos para el parto pretérmino. Es de llamar la atención que en el $82 \%$ de los casos, el embarazo fue interrumpido por vía abdominal, con el diagnóstico de ruptura prematura de membranas como el más frecuente, seguido de oligoamnios, sufrimiento fetal, embarazo múltiple, desprendimiento prematuro de placenta normoinserta, entre otros.

El peso de nacimiento está correlacionado con los días de estancia hospitalaria, a razón de menor peso tardará más tiem- po en alcanzar un peso óptimo, ya que la meta constante de crecimiento de acuerdo a las guías de práctica clínica basadas en evidencia, es obtener una ganancia de peso al menos como la del desarrollo intrauterino $(15 \mathrm{~g} / \mathrm{Kg} /$ día hasta el término). En nuestro estudio, la ganancia de peso promedio fue de $22.1 \pm 7.4 \mathrm{~g} /$ día. Tras una evolución satisfactoria, se decide el egreso que se realiza al momento de convertirse en conveniente para el desarrollo neonatal, que en promedio ocurrió a los $1754 \pm$ 185g. Solo en el 9.9\% $(n=7)$ de los casos, fue necesario el reingreso al servicio de CUPA I y un $2.8 \%(n=2)$ a CUPA II por no presentar datos de mejoría ni cumplir los objetivos de ganancia o presentar complicaciones.

Casi la mitad de los neonatos del MMC fueron alimentados $(49.2 \%, n=35)$ con leche materna complementada con fórmula para prematuro, en el $40.8 \%(n=29)$ se optó por la alimentación mediante fórmula para prematuros y en un porcentaje pequeño $(8.5 \%, n=6)$ los neonatos fueron alimentados con leche materna, como caso particular se administró leche de soya a un paciente $1.4 \%$. Si son conocidas las ventajas que tiene la leche materna, ${ }^{32-36}$ entonces debería de priorizarse la alimentación de todos los paciente del Programa Mamá Canguro en el Hospital de la Mujer de Aguascalientes, ya que precisamente el PMC la considera como uno de sus objetivos.

El 52\% de neonatos requirió medidas extras de reanimación, con el consecuente riesgo de lesión al sistema nervioso central irreversible y/o alguna otra afectación neurológica, por lo que es muy importante para estos infantes contar con la estimulación adecuada para su recuperación, estimulación que ofrece el Programa Mamá Canguro. ${ }^{30}$ 


\section{Conclusiones}

Los productos pretérmino y los neonatos de bajo peso al nacer continúan siendo una demanda que involucra tanto a obstetras, neonatólogos y personal especializado de enfermería. EI MMC representa una alternativa que la UNICEF ha promovido a nivel mundial como un método para proteger la integridad de los recién nacidos. Es necesario identificar las principales causas de parto pretérmino para que los médicos de primer nivel hagan la prevención o en su caso el tratamiento adecuado. Los hallazgos de este trabajo, nos hacen reflexionar acerca de la necesidad de continuar con esta línea de investigación, para realizar estudios prospectivos y comparativos que incluya un mayor número de neonatos, procedentes de diversas instituciones de nuestro estado.

\section{Bibliografía}

1. Gómez A, Pallás Alonso CR, Aguayo J. El método canguro, nutrición infantil. Acta Pediátrica Española, 2007;65(6):286-291

2. Torres J, Palencia D, Sánchez DM, García J, Rey H, Echandía CA. Programa Madre Canguro: primeros resultados de una cohorte de niños seguidos desde la unidad neonatal hasta la semana 40 de edad postconcepcional. Colombia Médica 2006; 3796101. Disponible en: http://www.redalyc.org/articulo.oa?id=28337203.

3. Villanueva Egan L., Contreras Gutiérrez A., Mauricio Pichardo Cuevas M., Rosales Lucio J; Perfil epidemiológico del parto prematuro; Revista de Ginecología y Obstetricia de México, 2008;76(9):542-548

4. Método Madre Canguro Guía Práctica, Ginebra, Organización Mundial de la Salud, 2004

5. Lawn J.E., Mwansa-Kambafwile J., Horta B. L., Barros F. C., Cousens S; Kangaroo mother care' to prevent neonatal deaths due to preterm birth complications; International Journal of Epidemiology 2010;39:i144-i154

6. Martínez H., La metodología Madre Canguro, Innovaciones para el desarrollo y la cooperación sur-sur, IDEASS Colombia.

7. Charpak N., Ruíz J. G., El Método Madre Canguro no es una alternativa al cuidado neonatal sino su complemento." APREM., Asociación de padres de niños prematuros, Madrid, España.

8. Whitley J. A; Rich, Bonnie L, A Double-Blind Randomized Controlled Pilot Trial Examining the Safety and Efficacy of Therapeutic Touch in Premature Infants. Advances in Neonatal Care: December 2008; 8(6):315-333

9.- Charpak N., Ruiz-Peláez J. G., Figueroa Z., Charpak Y. A Randomized, Controlled Trial of Kangaroo Mother Care: Results of Follow-Up at 1 Year of Corrected Age. PEDIATRICS, 2001;108(5):1072-1079

10. Réjean Tessier, Cristo M. B., StVelez S., Giron M., Nadeau L., Figueroa de Calume Z., Juan G. Ruiz-
Paláez J. G., Charpak N; Kangaroo Mother Care: A method for protecting high-risk low-birth-weight and premature infants against developmental delay; School of Psychology, Laval University, Laval, Qué., Canada, Kangaroo Mother Care Program, Children's Clinic, Colombian Social Security, Kangaroo Foundation, Bogotá, Colombia, Clinical Epidemiology Unit, Faculty of Medicine, Javeriana University, Bogotá, Colombia, June 2003

11. Rintaro Mori, Rajesh Khanna, Debbie Pledge, Takeo Nakayama; Meta-analysis of physiological effects of skin-to-skin contact for newborns and mothers, Pediatrics International,2010;52(2):161-170.

12. Feldman $R$, Eidelman $A$, Direct and indirect effects of breast milk on the neurobehavioral and cognitive development of premature infants. Developmental Psychobiology, 2003;43(2):109-119

13. AL Jefferies; Canadian Paediatric Society, Fetus and Newborn Committee. Kangaroo care for the preterm infant and family. Paediatr Child Health 2012;17(3):141-143.

14. Zhao J., Gonzalez F., Mu D. Apnea of prematurity: from cause to treatment, Eur J Pediatr 2011;170:1097-1105

15. Conde-Agudelo A, Belizán J, Diaz-Rossello J. Atención de madres canguro para reducir la morbilidad y la mortalidad en lactantes de bajo peso al nacer. Cochrane Database of Systematic Reviews 2011 Issue 3. Art. No.: CD002771. DOI: 10.1002/14651858. CD002771

16. Lawn J., Mwansa-Kambafwile J., Horta B., Barros F., Cousens S.; 'Kangaroo mother care' to prevent neonatal deaths due to preterm birth complications. International Journal of Epidemiology 2010;39:i144i154

17. Sari Goldstein Ferber S., Makhoul I. R., The Effect of Skin-to-Skin Contact (Kangaroo Care) Shortly After Birth on the Neurobehavioral Responses of the Term Newborn: A Randomized, Controlled Trial; Pediatrics, 2004;113(4):858 -865

18. Johnston C. C., Filion F., Campbell-Yeo M., Gou- 
let C., Bell L., McNaughton K., Byron J., Aita M., Finley G. A., Walker C; Kangaroo mother care diminishes pain from heel lance in very preterm neonates: A crossover trial; BMC Pediatrics 2008, 8:13 doi:10.1186/1471-2431-8-13

19. Gray L., Watt L., Blass E. M; Skin-to-Skin Contact Is Analgesic in Healthy Newborns; Pediatrics Vol. 105 No. 1 January 2000, Disponible en http://www.pediatrics.org/cgi/content/full/105/1/e14

20. Xiaomei Cong, Susan M. Ludington-Hoe, Stephen Walsh; Randomized Crossover Trial of Kangaroo Care to Reduce Biobehavioral Pain Responses in Preterm Infants: A Pilot Study, Biol Res Nurs April 2011;13(2):204-216.

21. Geeta Gathwala, Bir Singh, Jagjit Singh; Effect of Kangaroo Mother Care on physical growth, breastfeeding and its acceptability, Trop Doct October 2010;40(4):199-202

22. Somashekhar M. Nimbalkar, Neha S. Chaudhary, Keshardan V. Gadhavi, Ajay Phatak; Kangaroo Mother Care in Reducing Pain in Preterm Neonates on Heel Prick, The Indian Journal of Pediatrics, January 2013;80(1):6-10

23. Marín Gabriel M.A, Llana Martín I., A López Escobar A., Fernández Villalba E., Romero Blanco T., Touza Pol P; Randomized controlled trial of early skin-toskin contact: effects on the mother and the newborn, Acta Paediatrica, 2010;99(11):1630-1634.

24. Collados-Gómez L., Aragonés-Corral B. Contreras-Olivares I., García-Feced E., Vila-Piqueras $M$.

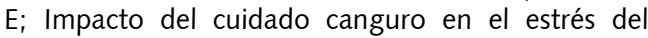
neonato prematuro, Enfermería Clínica Volumen 2011;21(2):69-74.

25. Castiblanco López N., Muñoz de Rodríguez L; Visión de las madres en el cuidado del hijo prematuro en el hogar, Enferm XXIX, 2011;1:120-129.

26. Lucía Gutiérrez L., Galleguillos D; Desarrollo del Vínculo de Apego en la díada Madre-Bebé prematuro, que practicaron el Método Madre Canguro en la Unidad de Neonatología del Hospital San José. Universidad Academia de Humanismo Cristiano, Chile septiembre 2011.

27. Arivabene JC., Rubio Tyrrell MA; Método madre canguro: vivencias maternas y contribuciones para la enfermería, Rev. Latino-Am. Enfermagem, 2010;18(2): [07 pantallas]
28. Goldenberg RL, Culhane JF, lams JD, Romero R. (0501-2008). «Epidemiology and causes of preterm birth.». Lancet (2008) 371:75-84.

29. Lizarazo-Medina JP., Juan M. Ospina-Díaz JM., Ariza-Riaño NE; Programa madre canguro: una alternativa y costo eficaz para la protección de los recién nacidos prematuros o con bajo peso al nacer, Rev. Salud Pública.2012;14(2):32-45.

30. Aplicación del Método Madre Canguro en Bebés Prematuros y de Bajo Peso en 4 Países de América Latina, Proyecto de USAID de Mejoramiento de la Atención en Salud Oficina Regional América Latina, Noviembre 2011, Disponible en http://www.healthynewbornnetwork.org/sites/ default/files/resources/Aplicando_Metodo_Madre_ Canguro_Nov2011.pdf

31 Guía de práctica clínica. Control prenatal con enfoque de riesgo. Disponible en www.cenetec.salud. gob.mx

32. William McGuire, Ginny Henderson, Peter W Fowlie "ABC of preterm birth Feeding the preterm infant" BMJ, 2004;329(7476):1227-1230.

33. Programa madre canguro en el hospital civil de Guadalajara/ Congreso internacional de lactancia materna/ Programa de intervención temprana y técnica canguro / Julio 2010. Disponible enhttp://www.cucs. udg.mx/archivosdeciencia/pdf/AdeC\% 202011\% 20 vol3num $1 \% 20$ CONGRESO $\% 20$ MEDICO $\% 20 \mathrm{HOS}$ PITALES\% 20CIVILES\% 202011.pdf

34. Nicoli Godón M; Revisión sistemática: Revisión sistemática: a influencia do Método Canguro sobre a lactancia materna en enonatos de alto risco. Universidade da Coruña, Facultade de Ciencias da Saúde, Mestrado en asistencia e investigación sanitaria, 2012 Disponible en http://hdl.handle.net/2183/9928

35. Conde-Agudelo A, Diaz-Rossello JL, Belizan JM. Método madre canguro para reducir la morbimortalidad en neonatos con bajo peso al nacer. Base de Datos Cochrane de Revisiones Sistemáticas 2007, Número

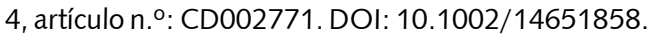
CD002771.

36. Groleau D., Ivone Evangelista Cabral I., Reconfiguring insufficient breast milk as a sociosomatic problem: mothers of premature babies using the kangaroo method in Brazil, Maternal \& Child Nutrition, 2009;5(1):10-24. 